\title{
Wide-banded NTC radiation: local to remote observations by the four Cluster satellites
}

\author{
P. M. E. Décréau ${ }^{1}$, S. Aoutou ${ }^{1}$, A. Denazelle ${ }^{1}$, I. Galkina ${ }^{1}$, J.-L. Rauch ${ }^{1}$, X. Vallières ${ }^{1}$, P. Canu ${ }^{2}$, S. Rochel Grimald ${ }^{3}$, \\ F. El-Lemdani Mazouz ${ }^{\mathrm{a}}$, and F. Darrouzet ${ }^{4}$ \\ ${ }^{1}$ Laboratoire de Physique et Chimie de l'Environnement et de l'Espace (LPC2E), Orléans, France \\ ${ }^{2}$ Laboratoire de Physique des Plasmas (LPP), Ecole Polytechnique, Palaiseau, France \\ ${ }^{3}$ Office National d'Études et de REcherches Aérospatiales (ONERA), The French Aerospace Laboratory, Toulouse, France \\ ${ }^{4}$ Belgian Institute for Space Aeronomy (IASB-BIRA), Brussels, Belgium \\ ${ }^{a}$ formerly at: Laboratoire ATmosphère, Milieux, Observations Spatiales (LATMOS), Guyancourt, France
}

Correspondence to: P. M. E. Décréau (pierrette.decreau@cnrs-orleans.fr)

Received: 2 June 2015 - Revised: 16 September 2015 - Accepted: 5 October 2015 - Published: 22 October 2015

\begin{abstract}
The Cluster multi-point mission offers a unique collection of non-thermal continuum (NTC) radio waves observed in the $2-80 \mathrm{kHz}$ frequency range over almost 15 years, from various view points over the radiating plasmasphere. Here we present rather infrequent case events, such as when primary electrostatic sources of such waves are embedded within the plasmapause boundary far from the magnetic equatorial plane. The spectral signature of the emitted electromagnetic waves is structured as a series of wide harmonic bands within the range covered by the step in plasma frequency encountered at the boundary. Developing the concept that the frequency distance $d f$ between harmonic bands measures the magnetic field magnitude $\boldsymbol{B}$ at the source $\left(d f=F_{c e}\right.$, electron gyrofrequency), we analyse three selected events. The first one (studied in Grimald et al., 2008) presents electric field signatures observed by a Cluster constellation of small size ( 200 to $1000 \mathrm{~km}$ spacecraft separation) placed in the vicinity of sources. The electric field frequency spectra display frequency peaks placed at frequencies $f_{s}=n d f(n$ being an integer), with $d f$ of the order of $F_{c \text { e }}$ values encountered at the plasmapause by the spacecraft. The second event, taken from the Cluster tilt campaign, leads to a 3-D view of NTC waves ray path orientations and to a localization of a global source region at several Earth radii $\left(R_{\mathrm{E}}\right)$ from Cluster (Décréau et al., 2013). The measured spectra present successive peaks placed at $f s \sim(n+1 / 2) d f$. Next, considering if both situations might be two facets of the same phenomenon, we analyze a third event. The Cluster fleet, configured into a constellation of large size $(\sim 8000$ to $25000 \mathrm{~km}$ spacecraft
\end{abstract}

separation), allows us to observe wide-banded NTC waves at different distances from their sources. Two new findings can be derived from our analysis. First, we point out that a large portion of the plasmasphere boundary layer, covering a large range of magnetic latitudes, is radiating radio waves. The radio waves are issued from multiple sources of small size, each related to a given $f s$ series and radiating inside a beam of narrow cone angle, referred to as a beamlet. The beamlets illuminate different satellites simultaneously, at different characteristic $f s$ values, according to the latitude at which the satellite is placed. Second, when an observing satellite moves away from its assumed source region (the plasmapause surface), it is illuminated by several beamlets, issued from nearby sources with characteristic $f_{s}$ values close to each other. The addition of radio waves blurs the spectra of the overall received electric field. It can move the signal peaks such that their position $f s$ satisfies $f s=(n+\alpha) d f$, with $0<\alpha<1$. These findings open new perspectives for the interpretation of NTC events displaying harmonic signatures.

Keywords. Magnetospheric physics (plasmasphere)

\section{Introduction}

The non-thermal continuum (NTC) terrestrial radio emissions have been observed, described and tentatively interpreted for about 40 years (Gurnett, 1975; Kurth et al., 1981; Etcheto et al., 1982; Gough, 1982; Morgan and Gurnett, 1991; Kasaba et al., 1998; Nagano et al., 2003; Décréau et 
al., 2004; Grimald et al., 2007, Décréau et al., 2013). NTC is an emission of weak to medium intensity, in the frequency range of about $10 \mathrm{kHz}$ to several $100 \mathrm{kHz}$, observed mostly inside the magnetospheric cavity, but also, for the highest frequencies of the spectra, escaping in the solar wind (Steinberg et al., 1989).

Although the precise generation mechanism has not yet been fully confirmed, it is widely accepted that the radio emission is created inside the plasmasphere boundary layer (PBL; defined in Carpenter and Lemaire, 2004), from the conversion of an intense seed electrostatic emission occurring at a frequency (plasma frequency, upper hybrid frequency or Bernstein mode frequency) characteristic of the local plasma (Jones, 1982; Morgan and Gurnett, 1991). The electrostatic to electromagnetic conversion is favoured by the locally strong gradient of density at the plasmapause boundary. The electrostatic emission results from the interaction of particles of $\mathrm{keV}$ energy, injected from the equatorial plasma sheet toward the Earth during active geomagnetic episodes, with the cold and dense population of the plasmasphere. This would happen predominantly in the dawn sector, where plasma sheet electrons are convected, a situation which has actually been suggested and validated by Gurnett and Frank (1976). This scenario, where incoming keV electrons near the Equator interact with the cold electrons of the plasmasphere, draws a picture where the sources of NTC waves are localized at the intersection of the geomagnetic equatorial plane with the PBL.

Studies of NTC waves by the multi-point Cluster constellation have allowed us to better understand and decipher the observed characteristics of spectral signatures of this radiation, and to translate them into genuine characteristics of the source region (Décréau et al., 2004; Masson et al., 2009; Grimald et al., 2009, 2011).

A novel type of radio wave, called wide-banded NTC, has been discovered by the Cluster constellation. Their main spectral and beaming properties have been described by Grimald et al. (2008). These waves, of weak intensity, are stemming from the PBL at medium latitudes, far from the magnetic Equator, in contrast with the scenario described above. They are infrequent (36 events in 4 years of Cluster observations), and mostly observed in the dawn sector (Grimald and Santolik, 2010). The modest magnitude of the observed electric field, and the fact that the waves are associated with the plasmapause layer and its density gradient, allows us to qualify them as NTC waves. The generation mechanism scenario described above should be revisited, since electrostatic waves observed away from the Equator are typically less intense than near the Equator. The general scheme, however, where an unstable electron distribution at medium latitudes creates locally seed electrostatic emissions converted into electromagnetic waves is still an obviously plausible scenario. It has been examined by Menietti and Yoon (2006) in the context of small-scale density structures, and by Grimald and Santolik (2010) who anal- ysed wave and particle properties observed by Cluster during a wide-banded NTC event.

This paper does not deal with NTC generation mechanism. It is devoted entirely to present and discusses observations of electric field waves measured during wide-banded NTC events by the unique tool offered by the multi-point Cluster mission. The underlying idea is to examine what is the topology of NTC sources and ray paths leading to the observations, such as to be able to better decipher the large number of signatures accumulated over years in Cluster data set, and to try to examine how a given local observation could be used as a clue to inform about the more global shape and state of the plasmasphere at that time.

Cluster, the first space mission designed to study the smallscale space plasma structures in three dimensions (Escoubet et al., 1997), is a constellation of four identical spacecraft (C1, C2, C3, C4). The Cluster satellites spin at a period of about $4 \mathrm{~s}$, with, under nominal conditions, all spin axes parallel to each other, oriented perpendicularly to the ecliptic plane. The satellites have been placed mostly in a regular tetrahedron configuration during the first years of the mission. In particular, a small-scale formation (satellite separation distances of the order of 100 to $1000 \mathrm{~km}$ near perigee) allows one to examine radio waves beaming properties by disentangling space and time. Later on, the spacecraft have been placed on several occasions in a multi-scale configuration, where three satellites form a large triangle, and the fourth one (most often $\mathrm{C} 4$ ) is placed at a variable distance from C3. Indeed, the two satellites share the same orbit and their separation distance is easily adjustable. When this distance is significant, the Cluster constellation offers a large receiving mesh, which can be illuminated at its different measuring nodes, by waves radiated from various parts of the PBL. When this distance is very small $(\sim 50 \mathrm{~km})$ another approach is made possible, whereby the pair of close spacecraft forms a single observatory. During one such configuration, in May 2008, the spin axis of C3 had been tilted by an angle of about $45^{\circ}$. For the first time, electric field measurements at the sensitivity available with long boom spinning antennas were accessible in 3-D. This new context opened the path to a new way of measuring electric field polarization properties.

We concentrate here on wave and density data obtained by the WHISPER (Waves of HIgh frequency and Sounder for Probing of Electron density by Relaxation) instrument (Décréau et al., 1997). WHISPER is a sounder which triggers resonances characteristic of the plasma within a region of a few kilometres surrounding the spacecraft. It measures local and radio waves at a good sensitivity, via an onboard fast Fourier transform (FFT) analyser, in the $2-80 \mathrm{kHz}$ range. WHISPER data are available from the four spacecraft during most of the Cluster mission, while measurements from the wide-band data (WBD) instrument (Gurnett et al., 1997), covering a similar frequency range with a better time and frequency resolution, have been by necessity restricted to selected time windows during the mission. No clear wide- 
banded NTC event has been pointed so far during these time intervals.

In the course of this paper, we present detailed observations of wide-banded NTC emissions obtained from three chosen events by the WHISPER instruments on board the Cluster spacecraft. We discuss their spectral characteristics, in particular the frequency distance between two successive peaks in frequency, $d f$, in view of the measured local electron gyrofrequency value, $F_{c e}$, and also the frequency band covered by the NTC emissions. We examine also, when possible, properties of the PBL crossed by the Cluster constellation during the events, as well as directivity properties attached to the NTC emissions. The underlying idea is to examine what is the topology of NTC sources and ray paths leading to the observations. This is facilitated by maps of boundaries and NTC observations in meridian planes, where isoFce surfaces are folded onto isoFce lines. Such maps have been used in Grimald at al. (2008) and in Décréau et al. (2013). We take the opportunity of this new analysis of NTC events previously published to correct an error which appears in the maps published in the two papers quoted above. This error concerns the shape of isoFce lines. It does not affect the substance and main results of the papers, but it has to be corrected.

We present in Sect. 2 the case event associated with the discovery of NTC of the wide-banded type, observed by Cluster in the close vicinity of associated sources, and in Sect. 3 an NTC event observed during the tilt campaign, when the Cluster constellation is placed at large distances from associated sources. In both cases, the particulars about corrections of published meridian maps are given. Section 4 presents an unpublished case, when the Cluster satellites observe various NTC waves, seen simultaneously at close distances and at larger distances from their likely sources. Section 5 is devoted to a general discussion based on the analysis of the three selected events. A summary of the main findings is given in Sect. 6 .

\section{A multi-point view: the 30 December 2003 event}

\subsection{General description}

The 30 December 2003 event has been analysed and described in Grimald et al. (2008). Several properties, which have been pointed in this study, are also present during other events of the same type, observed along similar orbits (Décréau et al., 2004; Grimald and Santolik, 2010). During such events, the Cluster spacecraft cross the plasmasphere within a narrow magnetic local time (MLT) sector and in the vicinity of the magnetic Equator (at magnetic latitudes (Mlat) within the interval $\pm 30^{\circ}$ ). Wide-banded NTC emissions are eventually observed in the outer plasmasphere region. In that case, a spectral analysis of the received signal displays peaks at values $f s$ which are exact multiples of the quantity $d f(\mathrm{fs}=n d f)$. The quantity $d f$ increases with the dis- tance of the observing spacecraft to the PBL, a behaviour common to all wide-banded NTC events observed during the first phase of the Cluster mission (Grimald et al., 2010). At plasmapause traversals, when they are still observed, the peak frequencies of the bands meet plasma frequencies close to harmonics of the local electron gyrofrequency $F_{c e}$ (Fig. 1 in Grimald and Santolik, 2010).

Figure 1a presents the frequency-time spectrogram of electric field variations measured by WHISPER on the inbound leg of the C3 Cluster satellite. This plot displays the electric field measured during passive operations, i.e. when the sounding part of the instrument is off, over the total frequency range covered by WHISPER. The quantity shown here is the potential difference between sensors of the Electric Field and Wave (EFW) instrument (Gustafsson et al., 1997), placed at the tips of one spinning long boom antenna, after a FFT performed onboard by WHISPER. It is expressed in $\mathrm{V}_{\mathrm{rms}} / \mathrm{Hz}^{1 / 2}$, and referred to as the signal $S$, square root of the electric field power spectral density, multiplied by the length $\mathrm{L}$ of the electric dipole $(88 \mathrm{~m})$. The colour code represents $S$ values in $\mathrm{dB}$ above $10^{-7} \mathrm{~V}_{\mathrm{rms}} / \mathrm{Hz}^{1 / 2}$. Three main harmonic bands of wide-banded NTC are observed over the 30 to $60 \mathrm{kHz}$ range, from about 08:30 to 08:46 UT.

\subsection{Density background}

The local plasma frequency $F_{\mathrm{p}}$ is displayed in Fig. 1a as a white dashed line. It is a combination of $F_{\mathrm{p}}$ values identified during active operations of the sounder, which occur here every $104 \mathrm{~s}$, and of $F_{\mathrm{p}}$ values identified during passive operations (Trotignon et al., 2010). The electron plasma density, $N_{\mathrm{e}}$, is related to $F_{\mathrm{p}}$ by the expression $N_{\mathrm{e}}=F_{\mathrm{p}}^{2} / 81$, where $F_{\mathrm{p}}$ is expressed in $\mathrm{kHz}$ and $N_{\mathrm{e}}$ in $\mathrm{cm}^{-3} . F_{\mathrm{p}}$ is observed well below the low frequency limit of the NTC emission range at the start of the event (08:25 UT). It increases slowly during the NTC episode (from 08:25 to 08:47 UT), then increases sharply, forming a PBL displaying several irregularities, involving successive positive and negative steep density gradients. As an example, the first density blob encountered, at 08:48 UT, is characterized by a positive density step of $25 \mathrm{~cm}^{-3}$ over a distance of $40 \mathrm{~km}$. Later on, from 08:50 to 08:54 UT, the irregularities encountered present less important density variations. The density profiles measured on the three other Cluster spacecraft are qualitatively similar, i.e. displaying strong irregularities, but the spacecraft are not close enough to each other to resolve the details of the observed density structures, as could be done with a close formation (Darrouzet et al., 2004). In the event discussed in this section, the largest separation between satellites (at 08:40 UT) is about $1000 \mathrm{~km}$ along the trajectory between $\mathrm{C} 1$ and $\mathrm{C} 4$, while $\mathrm{C} 2$ and $\mathrm{C} 3$, placed in between, are separated by about $200 \mathrm{~km}$ along a direction roughly perpendicular to the trajectory. This type of formation is referred to as a "line of pearls configuration". The outbound plasmapause (Fig. 1d), displays similar characteristics, i.e. a succession of density 
a)

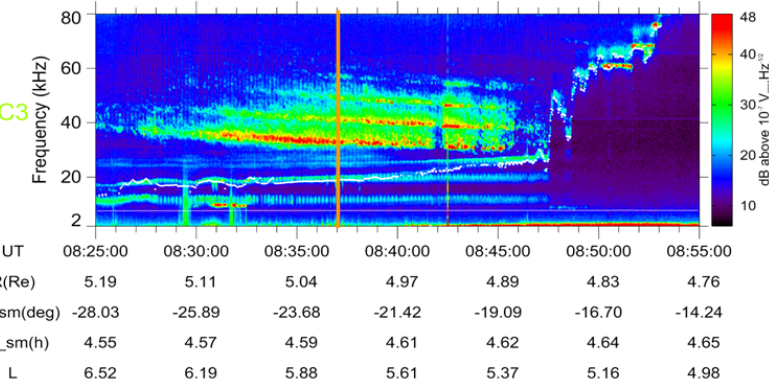

b)

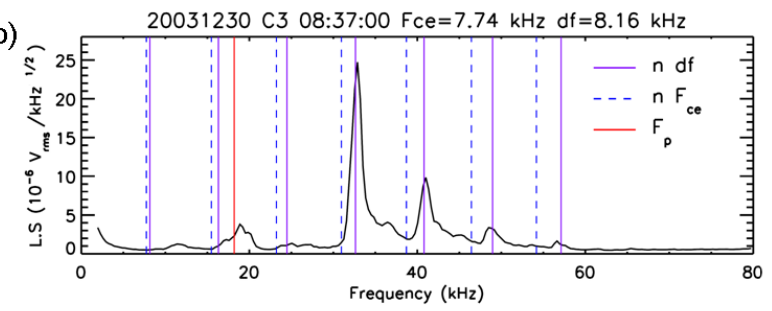$$
\text { F }
$$

c) $(\mathrm{kHz}$
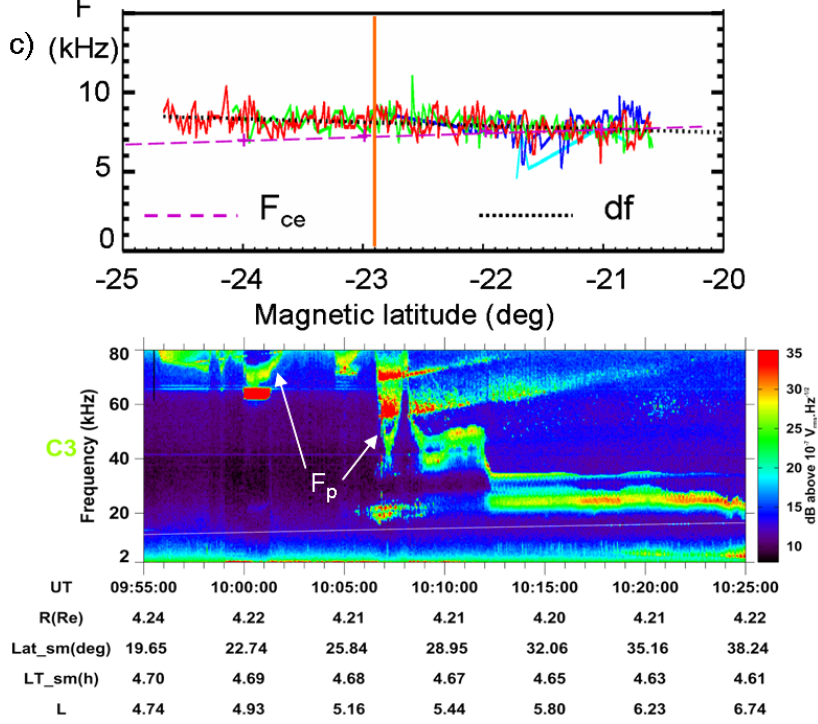

Figure 1. Spectral characteristics of the electric field measured by the $\mathrm{C} 3$ satellite of the Cluster constellation during the wide-banded NTC event of 30 December 2003. (a) Time-frequency spectrogram when $\mathrm{C} 3$ enters the plasmasphere. A white dashed line follows the plasma frequency $F_{\mathrm{p}}$ and a white solid line the electron gyrofrequency $F_{c e}$. (b) Individual frequency spectrum measured at 08:37:00 UT (orange line in panels a and c); the $n d f$ series is plotted as purple lines, the $n F_{c \text { e }}$ series as dashed blue lines. (c) Frequency distances $d f$ between two contiguous NTC peaks and $F_{c e}$ as a function of magnetic latitude (Mlat). (d) Time-frequency spectrogram when $\mathrm{C} 3$ leaves the plasmasphere. White arrows point to $F_{\mathrm{p}}$ spectral signatures.

blobs embedded in a PBL. Two NTC harmonic bands are observed by the spacecraft immediately after they leave the plasmasphere.

\subsection{Spectral characteristics of NTC beams}

As described above, the NTC bands are peaking at frequencies $f s$, harmonics of the characteristic frequency $d f$. The quantity $d f$ differs slightly from $F_{c e}$, with $d f>F_{c \mathrm{e}}$. Figure $1 \mathrm{~b}$ presents a single spectrum, signal amplitude vs. frequency, measured by WHISPER onboard C3. It is actually the average of five successive spectra measured every $1.7 \mathrm{~s}$ during a total duration of the order of $8 \mathrm{~s}$; the indicated time of the spectrum $(08: 37 \mathrm{UT})$ is the centre of the $8 \mathrm{~s}$ interval. This procedure is aimed at averaging out possible strong variations of the measured signal as the antenna rotates at the spin period of $4 \mathrm{~s}$. The electric field is actually significantly modulated with the antenna attitude, which allows us to derive signal directivity characteristics. Four frequency peaks equally spaced are present in the spectrum within the $30-60 \mathrm{kHz}$ band. Each one displays an asymmetric shape with respect to frequency, with a rising edge steeper than the falling edge. The magnitude of a peak is strongly decreasing with its position in frequency. Frequencies at multiple of $d f$ are indicated by purple vertical bars. Harmonics of $F_{c e}$ are indicated by dashed blue vertical bars. The $F_{\mathrm{p}}$ value, indicated by a red vertical bar, is identified by the lower cut off of a local emission, a criteria validated by sounding operations when they are available. This plot shows that the peaks are quasialigned with $n d f$ values and that $F_{c e}$ is smaller than $d f$ (of about $5 \%$ ).

When the spacecraft approaches the plasmapause from the Southern Hemisphere, i.e. when the altitude decreases and the Mlat increases (toward $0^{\circ}$ ), the characteristic interval $d f$ decreases. This behaviour is illustrated in Fig. 1c, reproduced from Grimald et al. (2008). Plots of frequency separation between two contiguous peaks are drawn as a function of the Mlat of C3; in red between the second and the first peak, in green between the third and the second peak, in dark blue between the fourth and the third peak, and in light blue between the fifth and the fourth peaks. All these plots are superimposed, confirming the relationship $f_{s}=n d f$. The variation of $d f$ with the Mlat of the view point is drawn as a black dotted line. The $F_{c \text { e }}$ local to the view point is derived from the fluxgate magnetometer (FGM) instrument (Balogh et al., 1997). It is plotted as a dashed purple line. Both curves meet at about $21^{\circ}$ Mlat, which happens a few minutes before $\mathrm{C} 3$ reaches the steep gradient in $F_{\mathrm{p}}$ marking the outside boundary of the plasmapause layer. The variation of $d f$ with the view point will be discussed further below.

\subsection{Multi-point view}

The observations from the four satellites of Cluster have been compared to each other in detail for this event (Grimald, 2007), allowing one to point out spatial properties at the scale of the Cluster constellation. The main result is reported in Grimald et al. (2008) and expressed as follows, when referring to time profiles of the electric field amplitude observed 


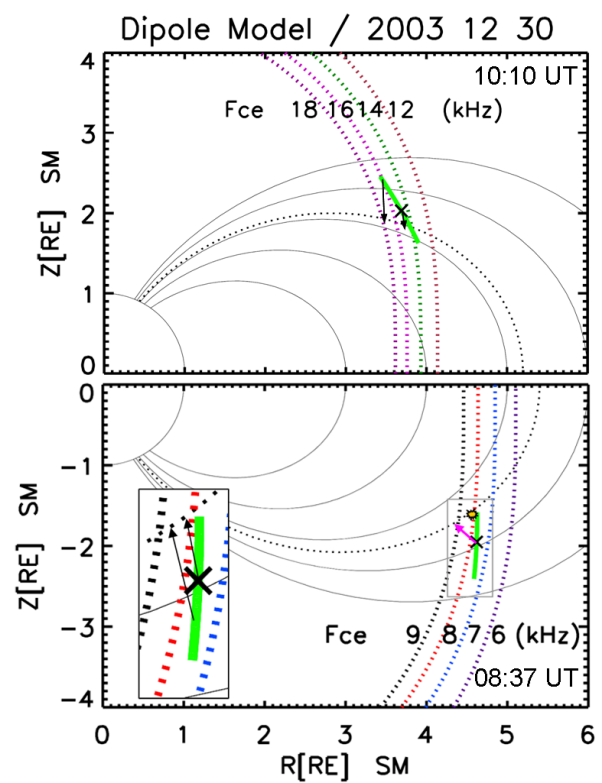

Figure 2. Meridian view of a dipole magnetic field model for the event of 30 December 2003. The Earth contour and L shells $(L=3$ to 7) are plotted in black. C3 orbit elements are shown as thick green lines. IsoFce curves (dashed squares) are drawn in various colours, according to the indicated $F_{c \text { e }}$ values. Plasmapause boundaries are shown as black dashed lines. Top: outbound orbit element, from 10:00 to 10:20 UT. The $X$ mark indicates C3 position at 10:10 UT. The black arrows join two successive positions of the satellite to the two associated source positions. Bottom: inbound orbit element, from 08:26 to 08:46 UT, including a detailed inset of the region of interest (sidebar on the left). The position of $\mathrm{C} 3$ at 08:37 UT is shown by an $X$ mark and the source position visible then by a yellow symbol. The pink arrow is aligned with an indicative orientation of the wave vector derived from spin modulation. Black arrows in the sidebar as in the top panel.

at a given frequency $(39.5 \mathrm{kHz})$ : “... all satellites display a similar behaviour, peaking times arranged in the order $\mathrm{C} 1$, $\mathrm{C} 2$ and $\mathrm{C} 3$, then $\mathrm{C} 4$, the exact ordering of spacecraft along the line of pearls configuration. It suggests a scenario where a stable beam, of limited cone angle, is illuminating just a part of Cluster orbit, reached by the different spacecraft one after another and crossed within a few minutes". This hypothesis is confirmed when displaying amplitude profiles vs. Mlat (Fig. 2 in the quoted paper). Each of the four latitudinal profiles of the electric field signal (expressed in $\mathrm{V}_{\mathrm{rms}} / \mathrm{Hz}^{1 / 2}$ ) displays a peak at $-21^{\circ}$ Mlat, of about $2^{\circ}$ width (the difference in Mlat when the signal intensity is half its peak value).

\subsection{Position of sources}

We derive NTC wave source positions via the approach proposed by Gough (1982) and developed in Grimald et al. (2008). This approach implies that sources related to harmonic signatures at $d f$ are part of the isoFce surface corresponding to the electron gyrofrequency $F_{c \mathrm{e}}=d f$. A series of sources are assumed to be activated at the intersection of this surface with a thin PBL of steep density gradient and where $F_{\mathrm{p}}$ verifies $F_{\mathrm{p}}=n d f$. In a 2-D view, the intersection of the isoFce surface with the PBL corresponds to a small area where the series of active sources are close enough to be considered as a single source of small size. In this paper, the position of the source radiating a $d f$ series is identified in the meridian map of a dipolar model of the Earth magnetic field at the intersection of the plasmapause $\mathrm{L}$ shell, derived from density profiles measured onboard Cluster, with the isoFce curve at $F_{c \mathrm{e}}=d f$. The source can be viewed from an observatory placed outside the plasmasphere if and when the source is beaming toward the observatory. Having determined the 2-D position of a source by intersecting the two curves indicated above, we deduce an empirical beam orientation from the segment plotted between the source and the view point.

The position of the source in the 3-D space, i.e. along the line of intersection of the PBL with the chosen isoFce surface, could in principle be obtained by ray tracing from the observatory toward the source. Indeed, the wave normal direction at a satellite can be estimated by signal modulation measured onboard, under the assumption of circular polarization (Décréau et al., 2013). A ray path could thus be derived, for instance in the frame of free propagation, and stopped when it crosses the isoFce surface at $F_{c e}=d f$, hopefully along its intersection with the plasmapause. In practice, this approach is subject to large uncertainties (except during the tilt campaign, as shown in the next section). As a consequence, we generally limit our analysis to a 2-D view.

We shall revisit now the map shown in Fig. 4 of Grimald et al. (2008) by correcting the error announced in the Introduction. A correct map is presented here in the bottom panel of Fig. 2 (the top panel of Fig. 2, which corresponds to a later time, is presented further down). It displays a meridian cut of magnetic field lines in a dipolar model and the orbit element of $\mathrm{C} 3$ during the interval 08:26 to 08:46 UT. It is labelled $R-Z$, where $R$ is the distance of a point from the dipole axis and $Z$ the distance from the equatorial magnetic plane. Because the dipolar model presents an axial symmetry with respect to the dipole axis, the configuration of $\mathrm{L}$ shells is identical at all local times. The same is true with isoFce surface shells which are drawn (dashed squares) in various colours, according to the indicated $F_{c e}$ values. This map allows one thus to visualize the position of a source according to the coordinates $L$ and $F_{c e}$, which can be chosen instead of $R$ and $Z$. For Fig. 4 of Grimald et al. (2008), L shells are correctly calculated, but isoFce lines are not, due to a confusion between the latitude and the co-latitude in one line of the software. This results in isoFce lines being flattened and too close to the Earth in the polar region. The model used in Fig. 2 of this paper considers a magnetic field intensity of 0.3 gauss on the Earth surface at the Equator (with $F_{c e}=840 \mathrm{kHz}$ ) and a value larger by a factor 2 at the poles. This results in $F_{c e}$ values $5 \%$ higher than the measured values (both by FGM and WHISPER sounder) along the studied orbit element of C3. 
Magnetic field intensities in the model presented in Fig. 2, bottom panel, are multiplied by a constant factor such that the modelled values fit the measured ones. The $L$ value chosen for the modelled plasmapause shell, $L=5.4$, corresponds to the outermost plasmapause $\mathrm{L}$ shell identified from the four density profiles measured during the four inbound plasmapause crossings. It is drawn with a black dotted line. The position of $\mathrm{C} 3$ at 08:37 UT is shown by an X mark. At this time, the $d f$ value is $8.16 \mathrm{kHz}$ (Fig. 1b). The position of the source in its meridian plane is, as stated above, at the intersection of the plasmapause $\mathrm{L}$ shell, with the isoFce line at $8.16 \mathrm{kHz}$. It is marked by a yellow symbol.

Directivity measurements of wide-banded emissions for this event have been presented in Grimald (2007), under the assumption of a circular polarization. This analysis yields wave vector orientations in the $X-Y$ plane of the geocentric solar ecliptic (GSE) coordinate system. It indicates that (1) all harmonic bands behave similarly, (2) the directivity angles derived at peak frequencies are significantly different from the values observed at other frequencies. In order to picture the ray path orientation in the SM (solar magnetic) coordinate system, we derive the 3-D coordinates of the wave vector at the observatory, using the approach described in Grimald et al. (2011). This wave vector, expressed in SM coordinate system, is represented in meridian and parallel planes of the dipole magnetic model by pink arrows (see Fig. 2 bottom panel but also Figs. 8 and 9). Note that the resulting orientations are only a crude indication of correct values, since the underlying assumption (circular polarization of electric field) is likely not fully satisfied (see Décréau et al. (2013) for an estimation of possible errors), and that measurement uncertainties are large. Nevertheless, Fig. 2 indicates that the wave vector points to a region not far from the source position obtained from $\mathrm{L}$ and $d f$ parameters. Projected onto a parallel plane, the wave vector makes an angle of about $25^{\circ}$ with the displayed meridian plane (placed at 4.6 MLT), pointing toward the Earth at an earlier MLT. This clue indicates that the sources of the observed waves are likely not placed in the $\mathrm{C} 3$ meridian plane.

\subsection{Proposed scenario}

In order to discuss the intriguing question raised above, about the opposite variations of the parameters $d f$ and $F_{c \text { e }}$ with the Mlat of observation (Fig. 1c), we propose an illustrative sketch in the sidebar shown in the bottom panel of Fig. 2. In this inset, the narrow beams (referred to as beamlets) intercepted by $\mathrm{C} 3$ at respectively $08: 32$ and 08:37 UT are represented by two black arrows joining the two $\mathrm{C} 3$ positions to the two sources respectively in view. The sources are placed at the intersections of the plasmapause L shell with the isoFce cuts corresponding respectively to $d f=8.7$ and $8.16 \mathrm{kHz}$ measured at the chosen times. The two beams are roughly parallel to each other. This indicates that similar conditions near sources lead to similar beaming properties.
It looks like the plasmapause surface emits beams at a given angle with its normal, an angle which is roughly independent from the emitted frequency, and which varies only slightly and progressively with the position of emission. Under the current geometric conditions, the sources visible from a distance are placed closer to the foot of the plasmapause magnetic field lines than the ones visible at a close approach, hence correspond to a larger $d f$ value.

The quantities $F_{c e}$ and $d f$ do not necessarily vary in opposition, since $d f$ is controlled by conditions at the source, while $F_{c e}$ is controlled by the satellite position. Indeed, when leaving the plasmasphere on its outbound leg, C3 observes both $d f$ and $F_{c e}$ increasing, as shown in Fig. 1d. This time-frequency spectrogram displays passive emissions measured by WHISPER. The $F_{\mathrm{p}}$ profile, pointed by white arrows, corresponds to the lower edge of banded electrostatic waves. C3 crosses an irregular PBL, then leaves the plasmasphere. Outside the external plasmapause boundary (crossed at 10:06:30 UT) wide-banded NTC waves, briefly blocked by a density blob at $\sim 10: 08 \mathrm{UT}$, are observed above $50 \mathrm{kHz}$. They are characterized by a frequency separation $d f$ equal to $F_{c e}$ at the boundary. The two bands of the NTC series are diverging with the distance to plasmapause; hence, $d f$ is increasing with time, and at the same time $F_{c e}$ (white line at the bottom of the spectrogram) increases. The meridian view associated with C3 outbound leg is shown in top panel of Fig. 2. The $L$ value at plasmapause is 5.2 (black dashed line). The C3 orbit element is plotted (green curve) from 10:10 to 10:20 UT. The dipole model has been corrected by a factor of $1 \%$ to fit $F_{c e}$ values measured along C3 orbit element. The orbit is more inclined with respect to isoFce lines (coloured dashed lines) than it is for the inbound leg. Two beams are traced as black arrows, joining C3 at 10:10 and 10:20 UT to the sources positions derived from $d f$ values, respectively 14.6 and $18.0 \mathrm{kHz}$. As observed in the Southern Hemisphere, the two beams are roughly parallel to each other and make a small angle with the plasmapause normal. We note that the illustrative sketches displayed in Fig. 2 are only factual ones. They do not suggest any physical mechanism controlling the beaming angles, in particular they do not explain why waves at different frequencies (the wide-banded NTC series) stemming from a source of small size should be grouped inside the same narrow beam.

\subsection{Complementary remarks}

As indicated by directivity measurements, the sources visible from a given view point are likely not in the same meridian plane than this point. A feature of the spectrogram in Fig. 1a is worth mentioning in this respect. The three narrow spectral lines observed at the upper frequency range between 08:50 and 08:53 UT could at first view be interpreted as local electrostatic sources of wide-banded NTC waves. They are indeed exact multiple of a given quantity $d f$. However, this quantity differs here slightly from the measured $F_{c e}$, 

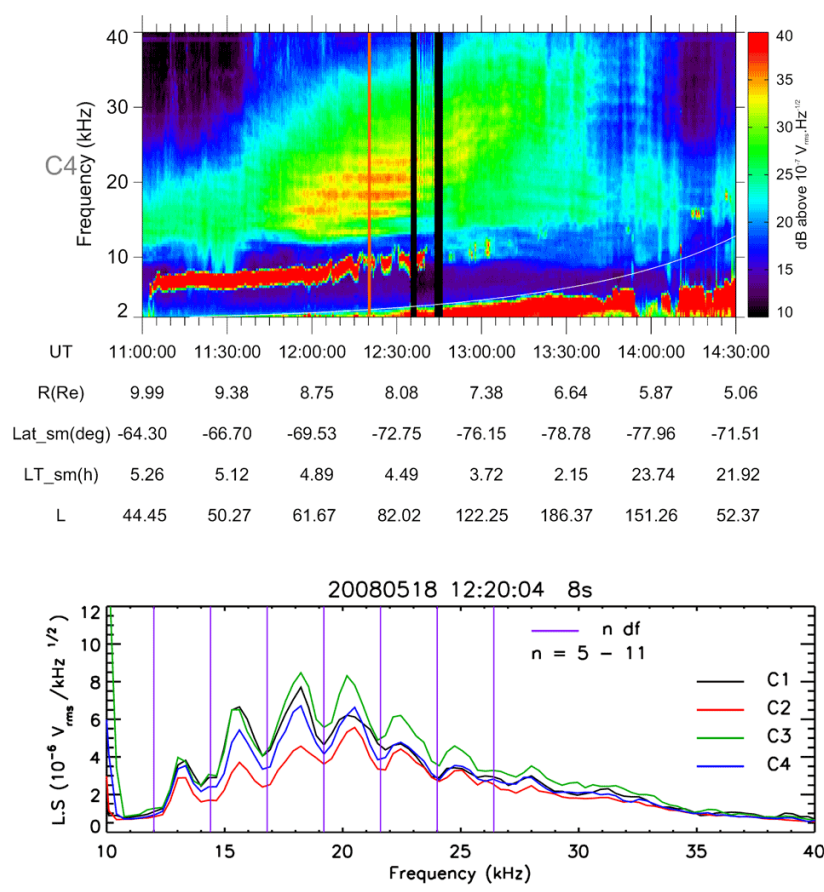

Figure 3. Spectral characteristics of the electric field measured by the Cluster constellation during the 18 May 2008 NTC event (after Décréau et al., 2013). Top: time-frequency spectrogram onboard C4. Bottom: Compared individual spectra at 12:20:04 UT (orange line in top panel) for the four Cluster spacecraft.

being $3 \%$ smaller. Furthermore, the observed lines stay at a constant frequency for significant time intervals (up to a minute) during which $F_{\mathrm{p}}$ fluctuates. Similarly on the outbound leg (Fig. 1d), a spectral line of high intensity is observed at 10:00 UT, below a dip in the $F_{\mathrm{p}}$ profile. It presents some similarity with the narrow spectral lines quoted above (displaying during about $1 \mathrm{~min}$ a tune at a constant frequency, close to a multiple of the local $F_{c \mathrm{e}}$ ). These signatures could be electromagnetic waves resulting from a more intense electrostatic instability, placed at some distance of the satellite, and participate to the generation mechanism of wide-banded NTC.

The comparison of wide-banded NTC waves observed respectively in the Southern and Northern hemispheres illustrates another interesting feature. When $\mathrm{C} 3$ moves outbound, the frequency range covered by the NTC bands is $\sim 50$ $80 \mathrm{kHz}$, as compared to a figure of $\sim 30-60 \mathrm{kHz}$ inbound. Similar observations are made by the three other Cluster spacecraft. We note that the plasmapause (at the sharpest density gradient) is placed, in all cases, at $F_{\mathrm{p}}$ values varying over the range of NTC bands observed respectively in one or the other hemisphere. The northern plasmapause crossings take place at altitudes closer to the foot of associated magnetic field flux tubes than at the inbound crossings, which is coherent with the presence of higher densities. We can in any event conclude that the proximity of a steep plasmapause gra- dient is one ingredient linked to wide-banded emissions, and it looks like the range of plasma frequency swept along this gradient delimits the overall range of NTC waves emitted, as if, below a given steepness, no waves are created.

\section{A 3-D view of NTC ray path: the 18 May 2008 event}

The case event on 18 May 2008 has been presented in detail in Décréau et al. (2013). It happens during the Cluster tilt campaign, when the pair of satellites $\mathrm{C} 3-\mathrm{C} 4$ is used as a single observatory, enabling to derive the geometric properties of a planar electromagnetic wave. It will be referred to as the tilt event further in this paper. The electric field time-frequency spectrogram recorded by $\mathrm{C} 4$ is shown on Fig. 3, top panel. It shows banded emissions from $\sim 11: 45$ to 13:00 UT which could belong to the wide-banded type. The single spectrum displayed in the bottom panel of Fig. 3 is similar to Fig. 6d in Décréau et al. (2013), but derived under the same conditions than all the single spectra presented in the current paper, by averaging spectra recorded during a short interval of $8 \mathrm{~s}$. Harmonics of the average frequency distance between peaks, $d f=2.4 \mathrm{kHz}$, are marked by vertical purple lines. All satellites see the same spectral signature at the same time, a signature characterized by peak positions at $f s \sim(n+1 / 2) d f$. Actually, the Cluster constellation is placed at a significant distance, about $5 R_{\mathrm{E}}$, from the source region derived in Décréau et al. (2013) study, such that all spacecraft are illuminated by the same beam. The spectrum in Fig. 3 differs qualitatively from a spectrum observed in typical widebanded NTC event in several aspects: (1) the peaks are not placed at exact multiple of the separation $d f$, (2) the ratio between peak amplitudes and the averaged amplitude is lower, and (3) the peak amplitudes do not decrease with the order of the harmonic.

The source position is derived via the approach of Gough (1982), in this case without the information about the size of the plasmasphere body. The measured parameter $d f$ is assumed to be equal to $F_{c e}$ at the source. The ray vector, which is derived with a good precision in 3-D from the orientation of the wave plane, is followed backwards under the assumption of free propagation, and stopped when the gyrofrequency from a model meets the measured frequency separation $d f$. This is illustrated in Fig. 9 of Décréau et al. (2013), which presents a meridian view of the magnetic field properties. This figure displays isoFce curves of the simple dipole model which are misplaced, due to the same error than the one affecting the meridian view presented in Grimald et al. (2008) and corrected above (Fig. 2, Sect. 2). Happily enough, the source positioning obtained via the erroneous simple dipole model has been discarded in the published study, as not physically coherent. Several 3-D models of the magnetospheric magnetic field, lacking axial symmetry, fit better the large data set of available spacecraft measurements (see Tsyganenko (2013) for a review). The 3-D model used in Décréau et al. (2013) yields a source position 


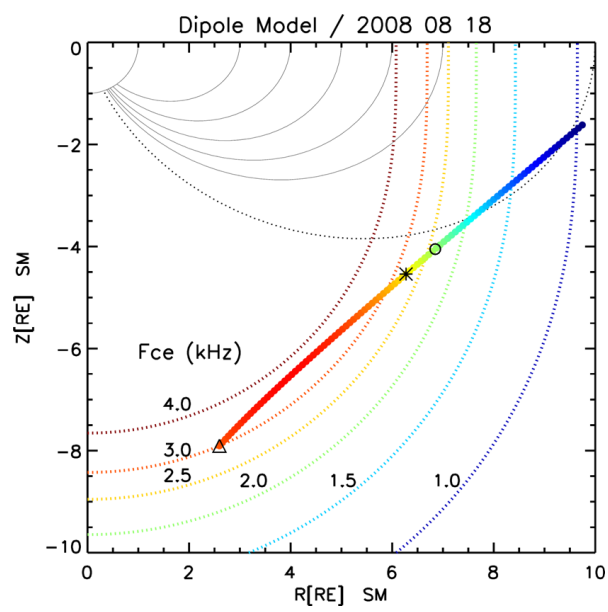

Figure 4. Meridian view, with $\mathrm{L}$ shells and isoFce contours of a simple dipolar model, as in Fig. 2. Chosen colours vary from brown $\left(F_{c \mathrm{e}}=4 \mathrm{kHz}\right)$ to dark blue $\left(F_{c \mathrm{e}}=1 \mathrm{kHz}\right)$, according to a blue-red colour table. An indicative plasmapause $L$ shell is drawn at $L=10$. The Cluster tilted pair at 12:20 UT is shown by a triangle. The ray path is traced backward under the free propagation approximation, forming a coloured thick line. The colours reflect local values taken by $F_{c \mathrm{e}}$ in the Tsyganenko model (same colour table than used for the isoFce contours in the dipolar model). The indicative position of the source is shown by an asterisk when using the Tsyganenko model, by an open circle when using the dipole model. The MLT value varies from 6.40 at source (in Tsyganenko model) to 4.65 at observatory.

at $X=-1.2 R_{\mathrm{E}}, Y=-6.45 R_{\mathrm{E}}$ and $Z=-4.1 R_{\mathrm{E}}$ in the GSE coordinate system. This position is placed near the $L=10$ shell, at medium Mlat $\left(\sim 35^{\circ}\right)$. It is considered to be correct and is discussed further in the paper.

A revisited meridian map of the simple dipolar model is presented in Fig. 4. Several isoFce lines are plotted with the dashed coloured curves, $F_{c e}$ values varying from 1 to $4 \mathrm{kHz}$. As in Fig. 2, they form ovoids, elongated along the dipole axis. The tilted pair position at the time of observation is marked by a black triangle. In this paper, we use the quantitative model of magnetic field derived by Tsyganenko (1987) with $\mathrm{Kp}=0$, a value representative of geomagnetic conditions for this case event. At the tilted pair position, the $F_{c e}$ value from the Tsyganenko model is higher by a factor of $5 \%$ than the measured value $(3 \mathrm{kHz})$ and the $F_{c e}$ value from the dipole model is smaller by a factor of $7 \%$ than the measured $F_{c e}$. In this revisited view, we have fitted both models to the measured value via adapted multiplying factors. The ray path, folded via axial rotations into the same meridian view, is plotted as a thick line, coloured according to the same blue-red table as used to represent the $F_{c \text { e }}$ values identifying the isoFce curves. The asterisk symbol indicates the source position, where the folded ray path reaches the point at $2.4 \mathrm{kHz}$ in the Tsyganenko model. The source position obtained via the simple dipole model, marked by an open circle, is placed close by. Assuming that this NTC source is placed at a PBL, we end up with a plasmasphere inflated up to a large $\mathrm{L}$ shell $(L \sim 11)$. This is probably due to specific geomagnetic conditions. In this case, more than 10 days at low activity are followed by a short increase of activity when the emissions are observed. The fact that sources are found away from the magnetic Equator, at medium Mlat, argues for identifying the observed emissions as wide-banded NTC emissions.

\section{A global view: the 26 August 2008 event}

The analysis of this case event, on 26 August 2008, forms the core of this paper. In a first part, we describe spectral characteristics obtained from data of the three Cluster spacecraft where the NTC waves are clearly present (Figs. 5 to 7). We question in particular the nature of the waves, electrostatic or electromagnetic. In a second part, we present and discuss global views derived from the spectral analysis (Figs. 8 and 9). These global views could be obtained thanks to the large dimension of the Cluster constellation during the event.

\subsection{Orbitography and density background}

The Cluster constellation has been placed at an orbit of high inclination angle at the start of the mission, and it was still the case in year 2003, during our first chosen case event. Later on, the orbit has slowly evolved. In year 2008, the orbit plane had an inclination angle of about $70^{\circ}$ and the perigee altitude had decreased from about 4 to $2.5 R_{\mathrm{E}}$ (see Fig. 1 in Lointier et al., 2013). In several orbits of August 2008, and later on, the Cluster satellites penetrate into the plasmasphere twice per orbit, in opposite local time sectors: (1) near perigee, crossing both hemispheres; and (2) on the outbound leg at larger geocentric distances, after flying over the northern polar cap. The second traversal happens only when the plasmasphere is inflated. In this case, the Cluster constellation grazes the outer part of the plasmasphere at medium latitudes.

The case event presented here occurs on 26 August 2008. The time of perigee is 12:45 UT (for C3, the satellite of reference). $\mathrm{C} 4$ crosses the dayside plasmasphere later, between $\sim$ 13:10 and 14:40 UT. WHISPER measurements allow one to identify a plasmapause boundary at $L \sim 7$ in both hemispheres on this first traversal. After flying over the polar cap, the formation reaches the night-side inner magnetosphere. $\mathrm{C} 2$ leads, followed by $\mathrm{C} 1$, then $\mathrm{C} 3$, and finally $\mathrm{C} 4$. Only C4, with its orbit being the closest to Earth, encounters briefly the plasmasphere near 16:50 UT, at $L \sim 7$. At this time, the Cluster tetrahedron is elongated, roughly along the orbit. The largest separation distance, between $\mathrm{C} 2$ and $\mathrm{C} 4$, is $25000 \mathrm{~km}$ and the smallest one, between $\mathrm{C} 1$ and $\mathrm{C} 3$, is $8000 \mathrm{~km}$. Figure 9 indicates the respective positions of the four Cluster spacecraft, projected into the magnetic equatorial plane, a view derived from OVT (Orbit Visualization 

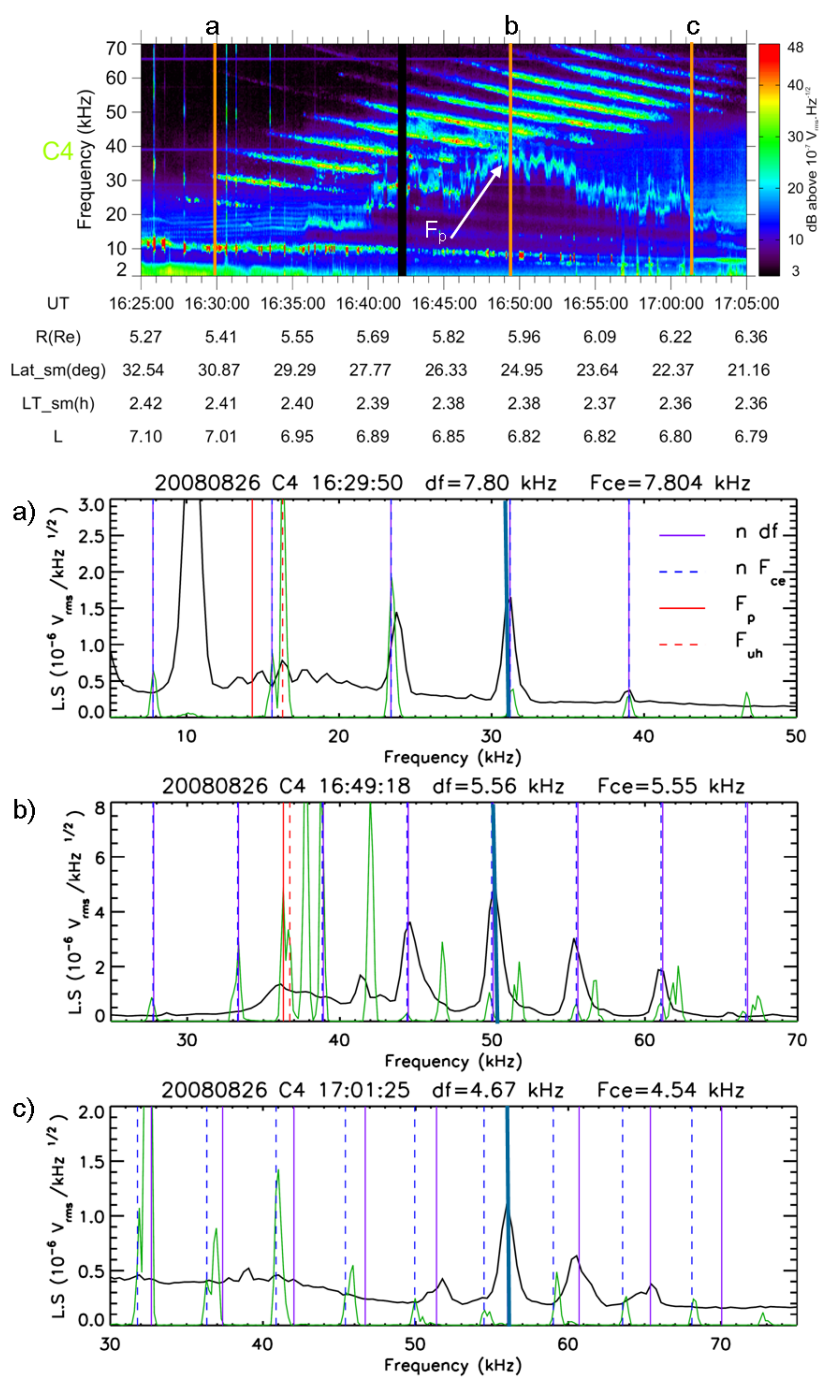

Figure 5. Spectral characteristics of the electric field measured by C4 during the 26 August 2008 event. Top: time-frequency spectrogram from 16:25 to 17:05 UT, orange lines indicate selected times of the spectra shown below. Bottom: individual spectra for the three selected times (a 16:29:50 UT, b 16:49:18 UT, c 17:01:25 UT); $d f$ and $F_{c \mathrm{e}}$ series plotted as in Fig. $1 ; F_{\mathrm{p}}$ and $F_{\text {uh }}$ positions are pointed by red segments; steel blue segments mark the frequency $F_{\mathrm{m}}$ of the main peak of the NTC series. Sounding spectra, recorded $8 \mathrm{~s}$ after indicated times, are plotted in green, with electric field magnitudes divided by a factor 100 .

Tool; http://ovt.irfu.se). Distances from this plane are indicated in Fig. 8 for orbit elements of $\mathrm{C} 4, \mathrm{C} 3$ and $\mathrm{C} 1$.

The brief encounter with the plasmasphere body is identified by an increase of $F_{\mathrm{p}}$ shown in the time-frequency WHISPER spectrogram of C4 on Fig. 5, top panel, by a white arrow. $F_{\mathrm{p}}$ corresponds to the lower edge of the banded emission at intensities coded in light blue. The $F_{\mathrm{p}}$ signature in natural emissions is present from $\sim 16: 36$ to $17: 05 \mathrm{UT}$. Its value increases from $\sim 15 \mathrm{kHz}$ at the start of the time interval, reaches $\sim 35 \mathrm{kHz}$ at 16:48 UT then decreases down
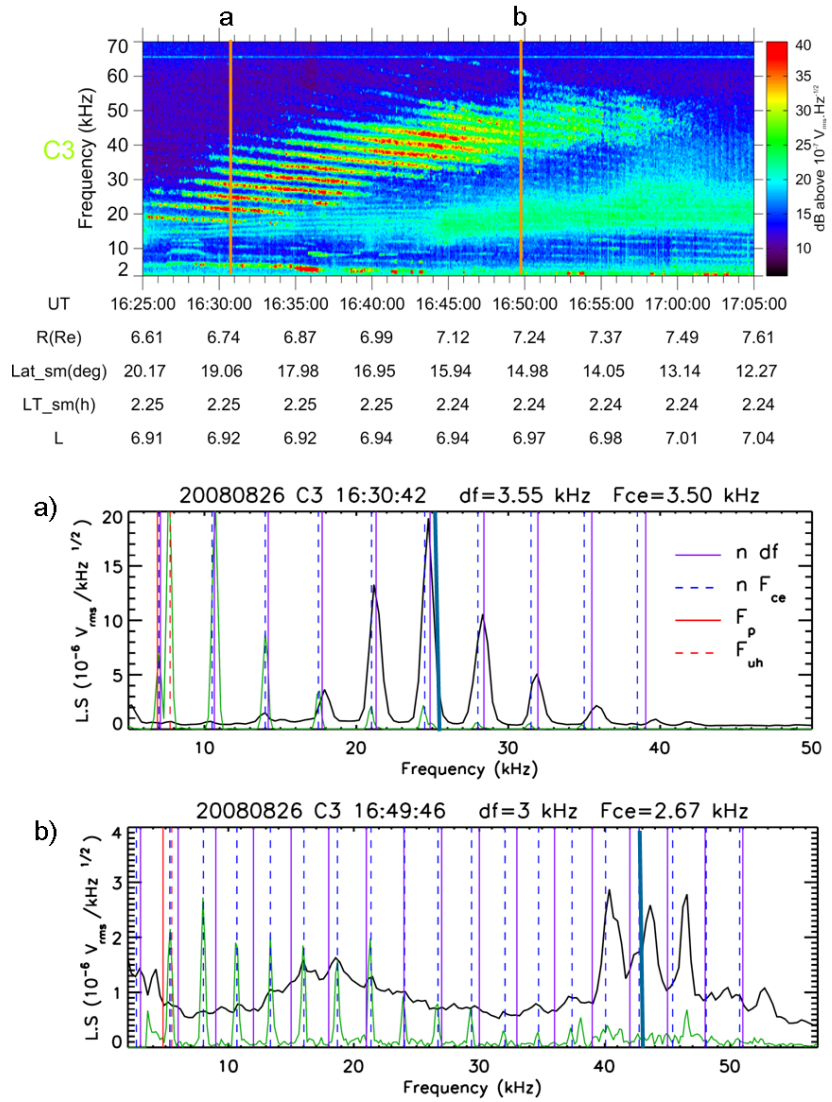

Figure 6. Spectral characteristics of the electric field measured by the C3 during the 26 August 2008 event. Top: time-frequency spectrogram from 16:25 to 17:05 UT, orange lines indicate selected times of the spectra shown below. Bottom: individual spectra for the two selected times (a 16:30:42 UT, b 16:49:46 UT) (presentation as in Fig. 5). Sounding spectra have electric field magnitudes divided by a factor of 50 and 10 , respectively.

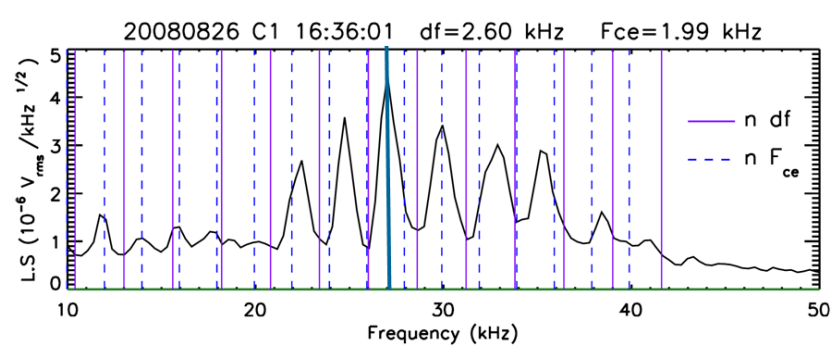

Figure 7. Frequency spectrum of the electric field measured by the $\mathrm{C} 1$ Cluster satellite during the 26 August 2008 event, at 16:36:01 UT (presentation as in Figs. 5 and 6).

to $\sim 15 \mathrm{kHz}$ at the end of the interval. The $F_{\mathrm{p}}$ time profile displays a great number of small-scale irregularities, but no local strong gradient over a significant range in $F_{\mathrm{p}}$ values. The PEACE (Plasma Electron And Current Experiment) instrument (Johnstone et al., 1997) confirms that only C4 encounters plasmaspheric electron populations on this part of 


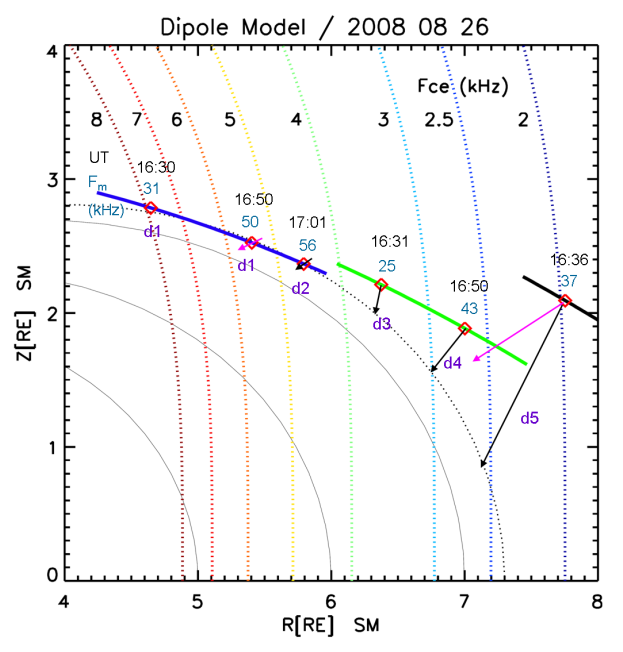

Figure 8. Meridian view during the 26 August 2008 event, with $\mathrm{L}$ shells and isoFce contours of a simple dipolar model, as in Figs. 2 and 4. An indicative plasmapause shell is drawn at $L=7.4$. The Cluster orbit elements during the event are displayed respectively in blue for $\mathrm{C} 4$, green for $\mathrm{C} 3$ and black for $\mathrm{C} 1$. Positions of the satellites at the times corresponding to individual spectra shown in Figs. 5 to 7 are marked by a red diamond symbol. Black arrows indicate backward ray paths of NTC radiation between view points and corresponding source positions. Pink arrows are aligned with indicative orientations of the ray path derived from directivity properties. Three pieces of information are given for each chosen satellite position: UT times, frequencies $F_{\mathrm{m}}$ of main frequency peaks (shown in Figs. 5 to 7) and labels (d1 to d5) referring to the distance between a source and the associated view point (see text in Sect. 4.4).

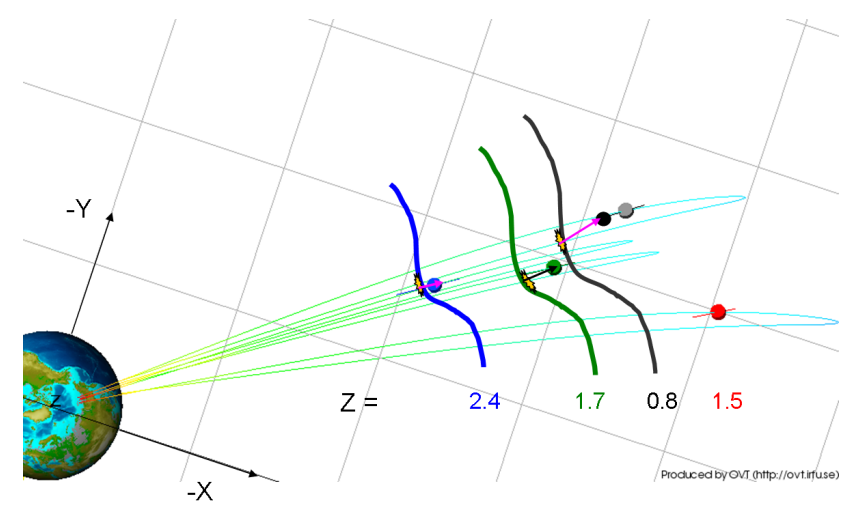

Figure 9. OVT view in $X O Y$ plane of SM coordinates, on $26 \mathrm{Au}$ gust 2008, at 16:50 UT. The Cluster satellites C1, C2, C3 and C4 are shown respectively as grey, red, green and blue cylinders, and intercepted field lines are drawn in light colours (red to blue, according to magnetic field magnitude). The black cylinder represents $\mathrm{C} 1$ at 16:36 UT. Cuts of the plasmapause at $Z$ positions (in $R_{\mathrm{E}}$ ) of NTC sources viewed by $\mathrm{C} 4$ and $\mathrm{C} 3$ at $16: 50 \mathrm{UT}$ and by $\mathrm{C} 1$ at 16:36 UT are sketched by thick lines respectively in blue, green and black. Possible sources are sketched by yellow stars. Pink arrows are aligned with indicative orientations of the ray path derived from directivity properties. the orbit and during the same time interval (measurements not shown).

\subsection{Spectral characteristics}

Wide-banded NTC emissions are observed on C4 during a time interval encompassing the episode of plasmasphere traversal described above, as shown in the top panel of Fig. 5. It is important to emphasize that the use of the term "time" in the following description is a language facility. What varies along the orbit is the view point of the observatory, while the physical process in action varies according to longer timescales. The NTC emissions consist of tones decreasing with the observation time, placed at all times at exact multiples of a frequency interval $d f$. The frequency range covered by the NTC series, about constant ( $\sim 25 \mathrm{kHz}$ width), is centred on a frequency which increases during the entire traversal from $31 \mathrm{kHz}$ at $16: 30 \mathrm{UT}$ to $56 \mathrm{kHz}$ at 17:01 UT. When C4 moves toward the plasmasphere, each band emits almost up to the time when its peak frequency meets the local $F_{\mathrm{p}}$. This is a similar behaviour than observed during the case event presented in Sect. 2. In contrast, when C4 moves away from the plasmasphere, the lower frequency limit of the range covered by the NTC bands is not related to any local property, suggesting that this frequency limit could be formed at some distance from the satellite, for instance by a nearby plasmaspheric blob of higher density.

The three bottom panels in Fig. 5 display each an instantaneous spectrum of electric field intensity vs. frequency at times marked by orange bars in the spectrogram on the top panel. As in Figs. 1 and 3 these spectra are averages within intervals of $8 \mathrm{~s}$ centred on the indicated times. The chosen time intervals are placed immediately prior to sounder operations (which occur a few seconds after the last passive acquisition). The spectral response of the sounded plasma is shown in green colour, after dividing the amplitudes by a factor of 100, allowing one to compare the frequency position of local resonances with frequencies of the NTC emissions. The explored medium is actually displaying clear resonances, which can be easily identified. The first combined spectrum (at 16:29:50 UT, Fig. 5a) displays three NTC peaks (black curve) between 20 and $40 \mathrm{kHz}$. The emission of high amplitude near $10 \mathrm{kHz}$ is probably an instrumental effect. It is discarded here. The average frequency distance between NTC peaks $f s$ defines the quantity $d f$, equal to $7.80 \mathrm{kHz}$. Harmonics of $d f$ are pointed by vertical bars in purple colour. The main peak in terms of intensity, referred to as $F_{\mathrm{m}}$, is underlined by a steel blue vertical bar. The active response (during sounding operations) displays resonances at multiple of $F_{c \mathrm{e}}$, pointing to a fundamental value $F_{c \mathrm{e}}=7.80 \mathrm{kHz}$. This value is identical (within measurement uncertainties of less than $1 \%$ ) to the ones deduced from FGM measurements of the magnetic field magnitude. The gyro harmonics are pointed in the figure by vertical lines in dashed blue colour. Since $d f$ and $F_{c e}$ are equal, marks of the two harmonic series are 
superimposed and cannot be distinguished from each other. The sounder triggers another resonance, of high amplitude, at $16.3 \mathrm{kHz}$ (dashed red vertical bar), which is identified as the upper hybrid frequency $F_{\mathrm{uh}}$ and leads to a plasma frequency $F_{\mathrm{p}}$ (red vertical bar) at $14.2 \mathrm{kHz} . F_{\mathrm{p}}$, significantly smaller than $f s$, is seemingly not related in any way to the spectral features of NTC emissions. This is the main argument leading to interpreting the waves as electromagnetic in nature. Finally, we note that, in contrast with the behaviour observed in Fig. 1 (for the first case event), the peak amplitude of the NTC series does not decrease as the frequency of the peak increases.

The second combined spectrum (at 16:49:18 UT, Fig. 5b) displays four NTC peaks, within the $43-63 \mathrm{kHz}$ frequency range. Again, the average frequency distance $d f$ equals the local measured $F_{c e}$ value. Again, the peak at smallest frequency is not the highest in amplitude. $F_{\mathrm{p}}$ is clearly identified, not only by a resonance at $F_{\text {uh }}$, but also by a series of Bernstein mode resonances $F_{Q}$, present inside $F_{c \text { e }}$ harmonic bands above $F_{\mathrm{p}}$ (Trotignon et al., 2010). Actually, the $F_{Q}$ resonances are peaking at higher intensities than the $F_{c \text { e }}$ harmonics, sometimes hardly visible in this plot (the vertical scale has been compressed). The local plasma frequency is not placed at this time in the vicinity of any peak of the NTC series, nor at any time during the event: the peaks are fading out a short time before the encounter with the plasmasphere. It is likely that the sources of the NTC emissions are not met by $\mathrm{C} 4$.

The third combined spectrum (at 17:01:25 UT, Fig. 5c) presents different properties than the two preceding ones. The NTC series exhibits four peaks within the 50$70 \mathrm{kHz}$ range, peaking roughly at multiples of a quantity $d f=4.67 \mathrm{kHz}$. The highest peak is the second one as ordered with increasing frequencies. The quantity $d f$ is significantly higher than $F_{c e}=4.54 \mathrm{kHz}$. The local resonances visible in this plot (which does not cover the plasma frequency, $\left.F_{\mathrm{p}} \sim 20 \mathrm{kHz}\right)$ are the $F_{c \mathrm{e}}$ series and the $F_{Q}$ series. This spectrum illustrates the two main spectral features which are observed when $\mathrm{C} 4$ leaves the vicinity of the plasmasphere: (1) the frequency separation $d f$ is not related any more to local magnetic properties, and (2) the lower cut off of the active frequency range is not related any more to local plasma frequencies. However, the bands are still peaking at multiples of a characteristic separation $d f$.

Observations from $\mathrm{C} 3$, placed at $\sim 1.8 R_{\mathrm{E}}$ from $\mathrm{C} 4$, are presented in Fig. 6, with a time-frequency spectrogram in the top panel, and two combined passive and active spectra in the lower panels. The presentation is the same than in Fig. 5. The wide-banded NTC emission observed in the $20-50 \mathrm{kHz}$ frequency range presents a high enough similitude with the emission observed from the $\mathrm{C} 4$ view point that we consider that they are closely related, but they have obviously different characteristics. The common features are (1) the presence of NTC banded series at frequencies decreasing with the observation time; (2) the frequency band covered is centred on a medium frequency which increases with time, roughly from 25 to $43 \mathrm{kHz}$; and (3) the lower edge of the frequency range displays some fluctuations at a few minutes timescale. In addition with the fact that one does not observe any signature of a plasmasphere traversal by $\mathrm{C} 3$, the main difference is that the characteristic frequency separation, $d f$, is of the order of $3 \mathrm{kHz}$, a value smaller of about a factor 2 than the value observed by $\mathrm{C} 4$.

The combined spectra shown in Fig. 6a, at 16:30:42 UT, yields $F_{\mathrm{p}}=7 \mathrm{kHz}$ and $F_{c \mathrm{e}}=3.50 \mathrm{kHz}$, smaller than the frequency separation $d f=3.55 \mathrm{kHz}$ of the observed NTC series. This is the same trend than observed in Fig. 5c, and will allow us in both cases to place the NTC sources closer to Earth than the observing satellites. The main peak of the NTC series is roughly in the middle of the total range, near $25 \mathrm{kHz}$. Figure $6 \mathrm{~b}$ displays combined spectra measured at 16:49:56 UT. In this case, $F_{\mathrm{p}} \sim 5 \mathrm{kHz}, F_{c \mathrm{e}}=2.67 \mathrm{kHz}$ and $d f=3 \mathrm{kHz}$, again larger than $F_{c \mathrm{e}}$. A new behaviour, compared to other spectra, is that the NTC peaks $f s$ are not anymore exact multiples of $d f$, but that they satisfy the relation $f s \sim(n+1 / 2) d f$ as for the second event (Sect. 3$)$.

C1 observes also faint banded emissions within the 15$40 \mathrm{kHz}$ range during the 16:25-16:50 UT time interval, but not in a steady way; the same is true for $\mathrm{C} 2$, displaying very faint and irregular features (not shown). Figure 7 shows a spectrum measured from $\mathrm{C} 1$ at 16:36:01 UT. The presentation is the same for the preceding plots, except for the sounder's response, which is not shown since its frequency range does not cover the plasma frequency, in this case smaller than $4 \mathrm{kHz}$. In this case, $d f$ is again significantly larger than $F_{c \mathrm{e}}\left(F_{c \mathrm{e}}=1.99 \mathrm{kHz}\right.$ and $\left.d f=2.60 \mathrm{kHz}\right)$. The NTC peak frequencies behave as in the case shown in Fig. 6b, they are not placed at exact multiples of $d f$, but in between.

In summary, the detailed analysis of spectral features observed from the Cluster satellites indicate that the widebanded NTC emissions are not related to local properties (except for the frequency separation, which closely meets the electron gyrofrequency when NTC bands are observed near the plasmasphere). These waves are thus very likely of electromagnetic nature. They are probably propagating over significant distances without being strongly attenuated. Electric field amplitudes observed on C3 for instance (Fig. 6a), are significantly higher than amplitudes observed on C4 (Fig. 5), despite the fact that the assumed source region, a plasmapause boundary, is not encountered, while it is crossed by $\mathrm{C} 4$ (noting that the observed waves cannot be directly compared, since the satellites do not cross the same beams).

\subsection{Meridian view}

The meridian view presented in Fig. 8 allows one to visualize the relative positions of NTC sources illuminating the different satellites along their trajectories. Like in Figs. 2 and 4,

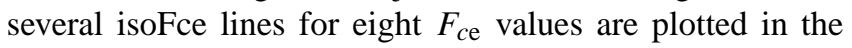


region of interest, in this case the northern side of plasmasphere, which is inflated up to an $\mathrm{L}$ shell of about 7.3 (black dotted line). The measured values of $F_{c e}$ are higher by a factor of $11 \%$ than the values deduced from the simple dipole model, which is adjusted in order that both measured and calculated values are equal. The orbit elements of $\mathrm{C} 4$ (blue thick line) and $\mathrm{C} 3$ (green thick line) are plotted for the time interval 16:20-17:06 UT. The orbit element of C1 (black thick line) covers the 16:25-16:45 UT time interval. C2 observes extremely faint and sporadic NTC emissions which are ignored in this study. All satellites travel away from the dipole axis, and toward the magnetic Equator. The positions of the satellites at the times corresponding to the individual spectra shown in Figs. 5 to 7 are marked by red diamond symbols. In order to better disentangle space and time when analysing this map we have indicated, for each selected position of a Cluster satellite, two pieces of information: the UT time (written in black) and the frequency $F_{\mathrm{m}}$ of the main peak of the NTC series (written in blue).

In contrast with the case event shown in Fig. 2 (30 December 2003), the C4 trajectory is directed roughly along an $\mathrm{L}$ shell, making large angles with isoFce lines. Consequently, the $F_{c \text { e }}$ parameter varies rapidly along the orbit. We can keep the interpretation given for the first case event. The sources at a given $d f$ signature are grouped within a steep density gradient at a given position in a meridian view. They are beaming within narrow cone angles. Under these conditions, the $\mathrm{C} 4$ satellite is illuminated by successive sources at different $d f$, in agreement with the encountered local $F_{c e}$ value, one at a time, explaining why peaks in the spectral signatures are well separated. Directivity measurements from modulation characteristics are unclear during this event, due to unfavourable sequencing periods of operations. One indicative value of the ray path orientation, derived in the SM coordinate system as explained in Sect. 2, is obtained near 16:50 UT. The ray path is close to the meridian plane (at an angle of $5^{\circ}$ westward) and directed as indicated by the small purple arrow. The black arrow associated with the third $\mathrm{C} 4$ position (at 17:01 UT) reflects the fact that at this time $d f$ is slightly larger than $F_{c \mathrm{e}}$ (Fig. 5c) indicating a source closer to Earth than the satellite.

The $\mathrm{C} 3$ trajectory (green) is placed significantly above the plasmapause $\mathrm{L}$ shell at $7.3 R_{\mathrm{E}}$. No directivity measurements are available during the event. The two black arrows are indicative of the ray path orientation. They join the satellite to the illuminating source, which is placed at the intersection of the isoFce line corresponding to the measured $d f$ marker with the $\mathrm{L}$ shell of the assumed plasmapause. The derived sources are closer to the Equator than the ones visible from $\mathrm{C} 4$. They are also placed at larger distances from the view points. C1 (black) travels still farther from Earth. The $d f$ marker associated with the measured spectrum shown in Fig. 7 points to a source position close to the Equator, away from the satellite by more than $1 R_{\mathrm{E}}$. A crude estimation of the (backwards) ray path orientation via spin modulation points to a direction at an angle of $\sim 20^{\circ}$ with the meridian plane, toward East, and is indicated by the pink arrow in the meridian plane.

The sketch in Fig. 9 completes the meridian view associated with this event. It displays the satellites and their magnetic field lines from a view point along the dipole axis at 16:50 UT and also at 16:36 UT for C1. The sources illuminating $\mathrm{C} 4$ and $\mathrm{C} 3$ at 16:50 UT and $\mathrm{C} 1$ at 16:36 UT are sketched by yellow symbols. They are placed at the plasmapause boundary, at the $Z$ values along the dipole axis indicated in the figure $\left(2.4 R_{\mathrm{E}}\right.$ for the source illuminating $\mathrm{C} 4,1.7 R_{\mathrm{E}}$ for the source illuminating $\mathrm{C} 3$ and $0.8 R_{E}$ for the source illuminating $\mathrm{C} 1$ ). These values correspond to the source positions derived in Fig. 8. Cuts of the plasmapause in planes parallel to the magnetic Equator at the $Z$ positions of sources are sketched by thick lines of the colour code related to each satellite (blue for $\mathrm{C} 4$, green for $\mathrm{C} 3$ and black for $\mathrm{C} 1$ ).

In conclusion, the view of the position of wide-banded NTC sources derived from the analysis of this event indicates that a large portion of the plasmapause is radiating during the same overall time interval, illuminating several satellites placed at large distances from each other. The associated beam cone angles have to be small enough to explain the spectral patterns observed, whereby a view point is illuminated according to a given $d f$, and the neighbouring orbit element illuminated according to a different $d f$. However, the width of cone angles is not precisely estimated in this analysis, neither in zenith angle (in a meridian plane) nor in longitude. Other observations are not well understood, such as the evolution with the view point of the lower edge of the emitted frequency range and of the central frequency of this range. We suggest, however, that a slight compression of the plasmasphere is taking place, resulting in a global drift of the plasma frequencies at the plasmapause density gradient. The geomagnetic indices are 0 or $0+$ during the $24 \mathrm{~h}$ time interval preceding the 26 August 2008, 12:00 UT, then 1- and 1 from 12:00 to 18:00 UT. Such a mechanism has to be confirmed.

\subsection{General discussion}

The compared analysis of the three case events reveals some similarities, despite the fact that they are observed from different perspectives:

1. A low geomagnetic activity prevails during a long time interval preceding each event, leading to a significant plasmasphere inflation. A moderate to low activity is observed during the event. The 1 day averaged Kp values are 1.3, 0.5 and 0.3 respectively for the December 2003 event, the May 2008 event, and the August 2008 event, while instantaneous $\mathrm{Kp}$ values are 1.3, 1.7 and 1 , respectively. Corresponding estimated plasmapause $\mathrm{L}$ shell values are 5.4, $\sim 11$, and 7.3, respectively. The estimated position of sources is placed in the 0-6 MLT sector for the three cases. 
2. Viewed from a small distance (December 2003 event and August 2008 event for C4) NTC waves are observed to radiate inside beams of small cone angles, along directions (when they are resolved, i.e. when $d f \neq F_{c e}$ ) not far from the normal to a plasmapause shell. Spin modulation characteristics indicate that the beams are likely making an angle with respect to the meridian plane of the dipole model at the longitude of the source, but this angle is not measurable precisely.

3. When observed, the PBL is very rough, displaying irregularities at many scales. The presence or formation of a plume type structure could play a role in the generation of wide-banded NTC waves. However, such structures are mainly placed in the afternoon sector (Darrouzet et al., 2008), in contrast with our observations. Note that one instance of plume structure in the afternoon sector associated with wide-banded NTC waves has been reported (Décréau et al., 2004; Darrouzet et al., 2006). In any case, the December 2003 case event favours a picture whereby localized steep density gradients inside an irregular PBL, are a necessary ingredient to the generation of wide-banded NTC emissions. Density profile patterns of this type are described in Horwitz et al. (1990). Referred to as type F profiles, they correspond to a fractional occurrence of about $5 \%$. This is coherent with the relative small occurrence of widebanded NTC events (Grimald et al., 2010).

One difficulty, when trying to elucidate the generation mechanism, is that during the limited number of widebanded NTC events available in Cluster data, no obvious traversal of intense electrostatic sources has yet been observed. The presence of candidate sources of moderate intensity has been identified a number of times (Grimald, 2007; Menietti and Yoon, 2006), in particular below or near local density dips of small scale. Some examples are also given in Sect. 2. None of them has been qualified as sources of wide-banded NTC waves. Likewise, the identification of electron distributions generating seed sources is yet to be done.

4. One important objective of this study is to discuss the evolution of wide-banded NTC wave signatures with the propagation distance along the ray path, aiming at answering the following question: are the harmonic waves studied during the tilt event the signature of a typical wide-banded NTC phenomenon - i.e. of the type discussed in Grimald et al. (2008) and in Grimald et al. (2010)? The August 2008 event analysed above in Sect. 4 yields a partial answer to that question, as developed below.

According to the interpretation presented in the meridian view displayed in Fig. 8, the distance (folded onto the meridian plane) from an observing satellite to the associated NTC source can be ranged from small to large as fol- lows: (1) unknown small distances for C4 before 17:00 UT, labelled as d1 in Fig. 8; (2) a distance d2 of about $400 \mathrm{~km}$ for C4 at 17:01 UT; (3) a distance d3 of about $1200 \mathrm{~km}$ for $\mathrm{C} 3$ at 16:30 UT; (4) a distance d4 of about $2500 \mathrm{~km}$ for C3 at 16:50 UT and (5) a distance $\mathrm{d} 5$ of about $1.4 R_{\mathrm{E}}$ for $\mathrm{C} 1$ at 16:36 UT.

The evolution in frequency ranges indicated in Fig. 8 shows clearly a time dependence of spectral signatures. Indeed, for the same time interval, from 16:30 to 16:50 UT, C4 and $\mathrm{C} 3$ observe a similar increase of the frequency $F_{\mathrm{m}}$ of the main peak, from 31 to $50 \mathrm{kHz}$ on $\mathrm{C} 4$, and from 25 to $43 \mathrm{kHz}$ on $\mathrm{C} 3$. This evolution is also clear in the spectrograms of Figs. 5 and 6 . The value measured on $\mathrm{C} 1$, at an intermediate time and frequency, completes the picture in a coherent way. The timescale of the increase in plasma frequencies at NTC sources (half an hour) is too short to reflect an increase of density due to plasmasphere refilling. It could be due to a plasmasphere compression, as suggested above (Sect. 4.3).

The evolution of wave intensity measured from the different selected positions does not seem to respond to any simple pattern either with space or time. However, one property is probably linked to the source to observer distance, namely the phasing of the peaks with exact multiples of the characteristic frequency separation. Actually, plots in Figs. $6 \mathrm{~b}$ and 7 display peak frequencies which do not satisfy the relationships $f s=n d f$ nor exactly $f s=(n+1 / 2) d f$, but they correspond better to the second one. The distances from source to view point, respectively $2500 \mathrm{~km}$ and $1.4 R_{\mathrm{E}}$ as indicated above, could be larger, when considering the ray path trajectory in longitude. We have tested a simple model, implying a sum of signals and could mimic a similar behaviour. The sketch in Fig. 10 helps to visualize the proposed scenario. The beam cone angles of two waves at neighbouring $d f$ markers are crossed at short distances from the sources by one satellite $(\mathrm{C} 4$, blue) and at larger distances by another satellite ( $\mathrm{C} 3$, green). In the case of $\mathrm{C} 4$, the beams are crossed one after the other. In the case of $\mathrm{C} 3$, the two beams are overlapping. The recorded electric field power density is the sum of two powers distributed slightly differently in frequency. This blurs out specific harmonic properties and can lead to peak distributions satisfying $f_{s} \sim(n+1 / 2) d f$ over given frequency ranges. We conclude that the specific spectral feature observed during the tilt event (Fig. 3) could indeed be produced by an ensemble of wide-banded NTC sources waves placed on the flank of the plasmasphere. However, there is still a difficulty to elucidate: the orientation of the ray path observed during the May 2008 event contrasts with orientations observed during the two other events. For both the December 2003 and the August 2008 events, the meridian views (Figs. 2 and 8 ) display wave vectors at sources oriented roughly away from Earth, whereas during the May 2008 event the wave vector, roughly tangent to the plasmapause, is directed toward the inner magnetosphere (Fig. 4). This peculiar behaviour is probably due to the presence of a shoulder (Pierrard and Lemaire, 2004), as proposed in Décréau et 


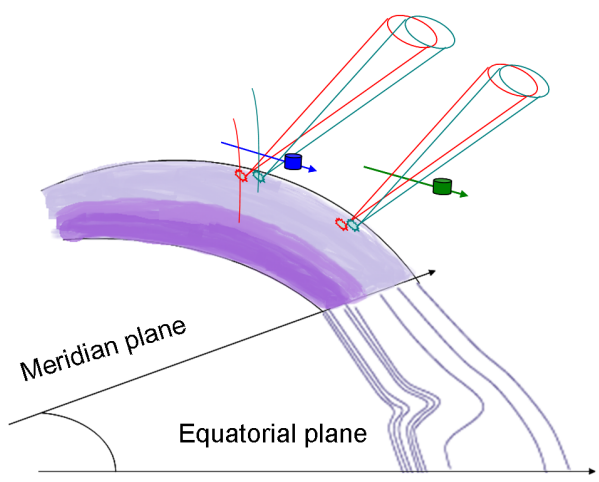

Figure 10. Sketch of NTC beams in 3-D, carrying each a specific spectral signature $d f$, illustrating how beams of similar cone angle might be crossed one at a time near sources (case of $\mathrm{C} 4$, in blue) and at the same time at larger distances (case of C3, in green).

al. (2013). In addition, this case event corresponds to a very inflated plasmasphere (plasmapause at $L \sim 11$ ), where plasmaspheric structures of complex shapes can be formed. Note that the meridian view does not allow one to appreciate the trajectory of the ray path in the third dimension (in azimuth). In this case, NTC waves travel from an MLT of 6.40 at the source to an MLT of 4.64 at the observatory.

\section{Summary and conclusion}

\subsection{Outline}

This paper presents morphological properties of beams radiated during NTC wave events characterized by wide-banded harmonic signatures. This study is motivated by the question of a possible link between, on one hand, NTC harmonic signatures when peaks in frequency satisfy $f s=n d f$ and, on the other hand, harmonic signatures when they satisfy $f s \sim(n+1 / 2) d f$.

The case event of December 2003 represents the standard according to which wide-banded NTC are identified. These NTC radiations are observed near the plasmasphere boundary layer, and their harmonic signatures satisfy $f s=n d f$. The typology of NTC harmonic signatures and of their evolution with the position of the view point is recalled in Sect. 2. This section describes also the approach used all along this paper, whereby the source of a punctual NTC signature (in space and time) is placed in a meridian map of the dipolar Earth magnetic field. This approach yields a 2-D view of the radiating part of the plasmapause. In this event, we have revisited the meridian view published in Grimald et al. (2008), related to the plasmapause crossing by Cluster in the Southern Hemisphere, and completed this view according to observations made at the plasmapause crossing in the Northern Hemisphere.

The case event of May 2008 represents the standard of NTC bands when harmonic signatures satisfy $f_{s} \sim(n+1 / 2) d f$. We have chosen this particular event because it is observed during the Cluster tilt campaign when the wave vector orientation at the view point is measured reliably, allowing one to place the source position in a meridian map via a simple ray tracing (under the assumption of free propagation). The revisited dipolar model presented in Sect. 3 indicates that, even in this case where the source and the view point are placed at large distances from the Earth ( $\sim 5$ to $8 R_{\mathrm{E}}$ ), a simple dipolar model allows one to identify roughly the source position (within $0.5 R_{\mathrm{E}}$ of the position obtained via the chosen Tsyganenko model).

This is in this context that the case event of August 2008 is analysed and presented in Sect.4. It has been chosen because the large separation distances between Cluster satellites allows one to examine NTC wave signatures both at small and large distances from the associated sources. We obtain several findings from the analysis and comparison of the three chosen case events.

\subsection{The raised interrogation about spectral signatures}

The August 2008 event shows an evolution of the overall spectral signature, from $f_{s}=n d f$, toward $f_{s} \sim(n+1 / 2) d f$ when the distance from source to observer is increasing. We interpret this behaviour by the superposition, at the largest distances, of several NTC beamlets at slightly different $d f$ characteristics, as discussed in Sect. 5. This leads to a positive answer to the interrogation about a possible link between the two observed types of spectral harmonic signatures. We conclude that a number of wave signatures structured in harmonic series with $f s \neq n d f$ could be interpreted as a superposition of waves emitted from the PBL at medium magnetic latitudes.

\subsection{Sectors of plasmapause shell surface radiating wide-banded NTC waves}

The topology of source regions observed in the Southern Hemisphere during the December 2003 event draws a picture of a plasmapause boundary emitting waves over a range of $\sim 8^{\circ}$ in Mlat, centred around $\sim-23^{\circ}$. The northern traversal of the plasmapause boundary, which happens a couple of hours after the southern traversal in the same MLT sector (within one degree in longitude) indicates sources which cover a range of $\sim 8^{\circ}$ in Mlat, centred around the parallel at $\sim 27^{\circ}$ latitude. In both hemispheres, the emission fades out when the view point leaves the indicated latitudinal range. In the absence of a 3-D perspective (due to the line of pearl configuration of the Cluster fleet), it is difficult to tell more about the overall dimension of radiating surface areas. The analysis of the August 2008 event yields a more global view of the latitudinal source distribution over the surface boundary at given times. The sources pointed in Fig. 8 are distributed continuously over Mlat covering the interval 6 to $32^{\circ}$, thus a range of $26^{\circ}$. At a time central to the event, the latitudinal 
range covered simultaneously amounts to $\sim 21^{\circ}$. The longitudinal size of the visible domain is small, due to the orbit configuration. Figure 9 indicates that the clearly exposed satellites (C1, C3 and C4) are placed within a longitudinal range of a few degrees. Taking account of the very faint emissions observed from $\mathrm{C} 2$, the total range amounts to $\sim 10^{\circ}$ in longitude.

\subsection{Findings relevant to generation mechanism}

Two findings have to be taken into account in a search for a relevant generation mechanism: beaming properties on one hand, and the morphology of the plasmapause surface on the other hand.

From studies in 2-D, the plasmapause surface seems to emit quasi-parallel beams of small cone angles where waves of a given $d f$ characteristic are concentrated. Each beamlet propagates the $d f$ signature attached to the source from which its stems. For the case events discussed in this paper when the wide-banded NTC are observed near the plasmasphere, the beam, folded onto the 2-D meridian map, makes a small angle with the normal to the plasmapause boundary.

Several observations obtained from studies at small scales indicate that an analysis in 3-D is necessary. One can quote (1) a beam propagating at a non zero angle with the meridian plane of the view point, as indicated by 3-D directivity estimations from spin modulation; (2) the presence of undulations of the plasmapause surface, identified either by NTC wave extinctions near plasmapause (Figs. 1a and 5, top panel) or by small size density blobs (Fig. 1d, 10:08 UT); and (3) the presence of potential electrostatic sources probably confined in azimuth (see Sect. 2.7 , complementary remarks).

\subsection{Perspectives}

A systematic study of wide-banded NTC events during the later phase of the Cluster mission (after year 2008) can be carried out to bring new findings about this phenomenon. The evolution of the orbit configuration will allow in particular to discover what is the dimension in longitude of the radiating part of the plasmapause surface, and hopefully discover the evolution of wide-banded NTC signatures from orbit elements encompassing the magnetic equatorial plane. Morphological studies can also help to discuss the evolution of wide-banded NTC events under dynamic geomagnetic conditions.

Acknowledgements. The authors thanks warmly the Cluster scientific and technical teams, in particular the Cluster operation team in ESOC, as well as the Cluster science mission centre in JSOC, for making the tilt campaign come true. They are grateful to the FGM team and the Cluster Active Archive for providing magnetic field data and to the WDC for Geomagnetism, Kyoto, for providing environmental data. CNES and ESA funding supported WHISPER instrument construction and support WHISPER data analysis.

The topical editor C.-P. Escoubet thanks O. Santolik and one anonymous referee for help in evaluating this paper.

\section{References}

Balogh, A., Dunlop, M. W., Cowley, S. W. H., Southwood, D. J., Thomlinson, J. G., Glassmeier, K. H., Musmann, G., Lühr, H., Buchert, S., Acuña, M. H., Fairfield, D. H., Slavin, J. A., Riedler, W., Schwingenschuh, K., Kivelson, M. G., and the Cluster magnetometer team: The Cluster magnetic field investigation, Space Sci. Rev., 79, 65-91, doi:10.1023/A:1004970907748, 1997.

Carpenter, D. L. and Lemaire, J.: The Plasmasphere Boundary Layer, Ann. Geophys., 22, 4291-4298, doi:10.5194/angeo-224291-2004, 2004.

Darrouzet, F., Décréau, P., De Keyser, J., Masson, A., Gallagher, D., Santolik, O., Sandel, B., Trotignon, J., Rauch, J., Le Guirriec, E., Canu, P., Sedgemore, F., André, M., and Lemaire, J.: Density structures inside the plasmasphere: Cluster observations, Ann. Geophys., 22, 2577-2585, doi:10.5194/angeo-222577-2004, 2004.

Darrouzet, F., De Keyser, J., Décréau, P. M. E., Gallagher, D. L., Pierrard, V., Lemaire, J. F., Sandel, B. R., Dandouras, I., Matsui, H., Dunlop, M., Cabrera, J., Masson, A., Canu, P., Trotignon, J. G., Rauch, J. L., and André, M.: Analysis of plasmaspheric plumes: CLUSTER and IMAGE observations, Ann. Geophys., 24, 1737-1758, doi:10.5194/angeo-24-1737-2006, 2006.

Darrouzet, F., De Keyser, J., Décréau, P. M. E., El LemdaniMazouz, F., and Vallières, X.: Statistical analysis of plasmaspheric plumes with Cluster/WHISPER observations, Ann. Geophys., 26, 2403-2417, doi:10.5194/angeo-26-2403-2008, 2008.

Décréau, P. M. E., Fergeau, P., Krasnosel'skikh, V., Lévêque, M., Martin, P., Randriamboarison, O., Sené, F. X., Trotignon, J. G., Canu, P., Mögensen, P. B., and WHISPER Investigators: WHISPER, a resonance sounder and wave analyser: performances and perspectives for the Cluster mission, Space Sci. Rev., 79, 157 193, doi:10.1023/A:1004931326404, 1997.

Décréau, P. M. E., Ducoin, C., Le Rouzic, G., Randriamboarison, O., Rauch, J.-L., Trotignon, J.-G., Vallières, X., Canu, P., Darrouzet, F., Gough, M. P., Buckley, A. M., and Carozzi, T. D.: Observation of continuum radiations from the Cluster fleet: first results from direction finding, Ann. Geophys., 22, 2607-2624, doi:10.5194/angeo-22-2607-2004, 2004.

Décréau, P. M. E., Kougblénou, S., Lointier, G., Rauch, J.-L., Trotignon, J.-G., Vallières, X., Canu, P., Rochel Grimald, S., ElLemdani Mazouz, F., and Darrouzet, F.: Remote sensing of a NTC radio source from a Cluster tilted spacecraft pair, Ann. Geophys., 31, 2097-2121, doi:10.5194/angeo-31-2097-2013, 2013.

Etcheto, J., Christiansen, P. J., Gough, M. P., and Trotignon, J.-G.: Terrestrial continuum radiation observations with GEOS-1 and ISEE-1, Geophys. Res. Lett., 9, 1239-1242, doi:10.1029/GL009i011p01239, 1982.

Escoubet, C. P., Schmidt, R., and Goldstein, M. L.: CLUSTER - Science and mission overview, Space Sci. Rev., 79, 11-32, doi:10.1023/A:1004923124586, 1997.

Gough, M. P., Non-thermal continuum emissions associated with electron injections: remote plasmapause sounding, Planet. Space Sci., 30, 657-668, doi:10.1016/0032-0633(82)90026-5, 1982. 
Grimald, S.: Etude de l'émission et de la propagation du Continuum terrestre à partir des données des satellites Cluster, Thèse de Doctorat, Université d'Orléans, France, 2007.

Grimald, S. and Santolik, O.: Possible wave modes of wideband nonthermal continuum radiation in its source region, J. Geophys. Res., 115, A06209, doi:10.1029/2009JA014997, 2010.

Grimald, S., Décréau, P. M. E., Canu, P., Suraud, X., Vallières, X., Darrouzet, F., and Harvey, C. C.: A quantitative test of Jones NTC beaming theory using CLUSTER constellation, Ann. Geophys., 25, 823-831, doi:10.5194/angeo-25-823-2007, 2007.

Grimald, S., Décréau, P. M. E., Canu, P., Rochel, A., and Vallières, X.: Medium latitude sources of plasmaspheric non thermal continuum radiations observed close to harmonics of the electron gyrofrequency, J. Geophys. Res, 113, A11217, doi:10.1029/2008JA013290, 2008.

Grimald, S., Foullon, C., Décréau, P. M. E., Le Rouzic, G., Suraud, X., and Vallières, X.: Modulation of NTC frequencies by Pc5 pulsations: experimental test of the generation mechanism and magnetoseismology of the emitting surface, J. Geophys. Res., 114, A11211, doi:10.1029/2009JA014270, 2009.

Grimald, S., El-Lemdani Mazouz, F., Foullon, C., Décréau, P. M. E., Boardsen, S. A., and Vallières, X.: Study of non-thermal continuum patches: wave propagation and plasmapause study, J. Geophys. Res., 116, A07219, doi:10.1029/2011JA016476, 2011.

Gurnett, D. A.: The Earth as a radio source: The nonthermal continuum, J. Geophys. Res., 80, 2751-2763, doi:10.1029/JA080i019p02751, 1975.

Gurnett, D. A. and Frank, L. A.: Continuum radiation associated with low-energy electrons in the outer radiation zone, J. Geophys. Res., 81, 3875, doi:10.1029/JA081i022p03875, 1976.

Gurnett, D. A., Huff, R. L., and Kirchner, D. L.: The WideBand Plasma Wave Investigation, Space Sci. Rev., 79, 195-208, doi:10.1023/A:1004966823678, 1997.

Gustafsson, G., Boström, R., Holback, B., Holmgren, G., Lundgren,A., Stasiewicz, K., Ahlen, L., Mozer, F. S., Pankow, D., Harvey, P., Berg, P., Ulrich, R., Pedersen, A., Schmidt, R., Butler, A., Fransen, A. W. C., Klinge, D., Thomsen, M., Fälthammar, C. G., Lindqvist, P.-A., Christenson, S., Holtet, J., Lybekk, B., Sten, T. A., Tanskanen, P., Lappalainen, K., and Wygant, J.: The electric field and wave experiment for the Cluster mission, Space Sci. Rev., 79, 137-156, doi:10.1023/A:1004975108657, 1997.

Horwitz, J. L., Comfort, R. H., and Chappell, C. R.: A statistical characterization of plasmasphere density structure and boundary locations, J. Geophys. Res., 95, 7937-7947, doi:10.1029/JA095iA06p07937, 1990.

Jones, D.: Terrestrial myriametric radiation from the earth's plasmapause, Planet. Space Sci., 30, 399-410, doi:10.1016/00320633(82)90046-0, 1982

Johnstone, A. D., Alsop, C., Burge, S., Carter, P. J., Coates, A. J., Coker, A. J., Fazakerley, A. N., Grande, M., Gowen, R. A., Gurgiolo, C., Hancock, B. K., Narheim, B., Preece, A., Sheather, P. H., Winningham, J. D., and Woodliffe, R. D.: Peace: A plasma electron and current experiment, Space Sci. Rev., 79, 351-398, doi:10.1023/A:1004938001388, 1997.

Kasaba, Y., Matsumoto, H., Hashimoto, K., Anderson, R. R., Bougeret, J.-L., Kaiser, M. L., Wu, X. Y., and Nagano, I.: Remote sensing of the plasmapause during substorms: Geotail observation of nonthermal continuum enhancement, J. Geophys. Res., 103, 20389-20405, doi:10.1029/98JA00809, 1998.
Kurth, W. S., Gurnett, D. A., and Anderson, R. R.: Escaping nonthermal continuum radiation, J. Geophys. Res., 86, 5519-5531, doi:10.1029/JA086iA07p05519, 1981.

Lointier, G., Darrouzet, F., Décréau, P. M. E., Vallières, X., Kougblénou, S., Trotignon, J. G., and Rauch, J.-L.: Refilling process in the plasmasphere: a 3-D statistical characterization based on Cluster density observations, Ann. Geophys., 31, 217-237, doi:10.5194/angeo-31-217-2013, 2013.

Masson, A., Santolik, O., Carpenter, D. L., Darrouzet, F., Décréau, P. M. E., Green, J. L., Grimald, S., El-Lemdani Mazouz, F., Moldwin, M. B., and Nemec, F.: Advances in plasmaspheric wave research with CLUSTER and IMAGE observations, Space Sci. Rev., 145, 137-191, doi10.1007/s11214-009-9508-7, 2009.

Menietti, J. D. and Yoon, P. H.: Plasma waves and fine structure emission bands within a plasmapause density cavity source region, Geophys. Res. Lett., 33, L15101, doi:10.1029/2005GL025610, 2006

Morgan, D. D. and Gurnett, D. A.: The source location and beaming of terrestrial Continuum radiation, J. Geophys. Res., 96, 95959613, doi:10.1029/91JA00314, 1991.

Nagano, I., Wu, X.-Y., Takano, H., Yagitani, S., Matsumoto, H., Hashimoto, K., and Kasaba, Y.: Remote sensing the magnetosheath by the spin modulation of terrestrial continuum radiation, J. Geophys. Res., 108, 1224, doi:10.1029/2002JA009691, 2003.

Pierrard, V. and Lemaire, J. F.: Development of shoulders and plumes in the frame of the interchange instability mechanism for plasmapause formation, Geophys. Res. Lett, 31, L05809, doi:10.1029/2003GL018919, 2004.

Steinberg, J. -L., Hoang, S., and Lacombe, C.: Propagation of terrestrial kilometric radiation through the magnetosheath: ISEE-3 observations, Ann. Geophys., 7, 151-160, 1989, http://www.ann-geophys.net/7/151/1989/.

Trotignon, J.-G., Décréau, P. M. E., Rauch, J. L., Vallières, X., Rochel, A., Kougblénou, S., Lointier, G., Facskó, G., Canu, P., Darrouzet, F., and Masson, A.: The whisper relaxation sounder and the Cluster active archive, in: The Cluster Active Archive, Studying the Earth's Space Plasma Environment, edited by: Laakso, H., Taylor, M. G. T. T., and Escoubet, C. P., Astrophysics and Space Science Proceedings, Springer, Berlin, Germany, 185208, doi:10.1007/978-90-481-3499-1_12, 2010.

Tsyganenko, N. A.: Global quantitative models of the geomagnetic field in the cislunar magnetosphere for different disturbance levels, Planet. Space Sci., 35, 1347-1358, doi.10.1016/003260633(87)90046-8, 1987.

Tsyganenko, N. A.: Data-based modelling of the Earth's dynamic magnetosphere: a review, Ann. Geophys., 31, 1745-1772, doi:10.5194/angeo-31-1745-2013, 2013. 